\title{
ESTUDO DO INSETICIDA CARBOFURANO EM SOLO E SEDIMENTO DE ÁREA DE PRODUÇÃO DE ARROZ IRRIGADO E CONTROLE DO GORGULHO AQUÁTICO ORYZOPHAGUS ORYZAE, TAUBATÉ, SÃO PAULO, BRASIL
}

\author{
M.R. Santiago-Moreira ${ }^{1}$, J.L.N. Mucci ${ }^{2}$, C.H.P. Ciscato ${ }^{1}$, S.H. Monteiro ${ }^{1}$, R.B. Abakerli ${ }^{3}$ \\ ${ }^{1}$ Instituto Biológico, Centro de Pesquisa e Desenvolvimento de Proteção Ambiental, Av Cons. Rodrigues \\ Alves, 1252, CEP 04014-002, São Paulo, SP, Brasil. E-mail: santiago@biologico.sp.gov.br
}

\section{RESUMO}

\begin{abstract}
Resíduos do inseticida e nematicida carbofurano e de seu principal metabólito 3-hidroxicarbofurano foram averiguados em tabuleiros de arroz irrigado e em áreas adjacentes para controle do gorgulho aquático da espécie Oryzophagus oryzae Lima, 1936. As amostras foram coletadas no período de 6/9/1999 a 4/5/2000 e analisadas por cromatografia a líquido de alta eficiência (HPLC) utilizando-se detector de fluorescência. Não foram detectados resíduos de carbofurano e de seu metabólito 3-hidroxicarbofurano dentro dos limites de determinação do método.
\end{abstract}

PALAVRAS-CHAVE: Resíduos de carbofurano, solo, sedimento, Oryzophagus oryzae, gorgulho aquático do arroz.

\section{ABSTRACT}

STUDY OF THE INSECTICIDE CARBOFURAN IN THE SOIL AND SEDIMENT OF PADDY RICE FIELDS WITH CONTROL OF THE WEEVIL ORYZOPHAGUS ORYZAE, IN TAUBATÉ, STATE OF SÃO PAULO, BRAZIL. The presence of residues of the insecticide and nematicide carbofuran, used for weevil (Oryzophagus oryzae Lima 1936) control, and its principal metabolite 3-hydroxycarbofuran were evaluated in soil and sediment plots from paddy rice fields and adjacent areas. The samples were analyzed using a high-performance liquid chromatography and fluorescence detector. Carbofuran and 3-hydroxycarbofuran residues were not detected in any sample in amounts above the method's detection threshold.

KEY WORDS: Residues of carbofuran, soil, sediment, Oryzophagus oryzae, weevil.

A agricultura moderna utiliza diversos insumos agrícolas, tais como fertilizantes, sementes melhoradas e transgênicas, máquinas agrícolas com alta tecnologia e os agrotóxicos, que podem levar a um comprometimento da qualidade dos alimentos e do meioambiente devidoà permanência deseus resíduos.

Na cultura do arroz irrigado por inundação no Vale do Paraíba, um dos principais agrotóxicos utilizados é o carbofurano (BRASIL, 2010). Este inseticida é aplicado no tabuleiro inundado para controle dos gorgulhos aquáticos. O carbofurano, inseticida e nematicida, do grupo químico dos carbamatos, é um composto sistêmico, que age por contato e ingestão. É utilizado no controle de um largo espectro de pragas agrícolas.

O carbofurano é pouco persistente e tende a se degradar rapidamente sob condições de solo inundado. Jury; GHodrati (1989), citados por Trabue (1997), verificaram que a persistência do carbofurano em solos foi moderada, exibindo uma meia vida média de 40 dias. Conforme JiNHE (1989), em ecossistemas de plantio de arroz conjugado com a criação de peixes, o desaparecimento de resíduos de carbofurano tanto em água como em solo de tabuleiro depende do método de aplicação do agrotóxico, como por exemplo a lanço ou incorporado.

As características de adsorção e dessorção que determinam o movimento de agrotóxicos através do perfil do solo, sua biodisponibilidade, degradação microbiana e persistência dependem das propriedades do solo, tais como conteúdo de matéria orgânica, conteúdo de argila e das propriedades físico-químicas do agrotóxico, tamanho e forma das partículas, solubilidade em água, valores de coeficiente de partição octanol-água (Kow) e polaridade (LaLAH; Wandiga, 1996).

FARAHANI et al. (2008) estudaram o movimento descendente de carbofurano na Malásia, usando

${ }^{2}$ Universidade de São Paulo, Faculdade de Saúde Pública, Departamento de Saúde Ambiental, São Paulo, SP, Brasil. ${ }^{3}$ UNICAMP, Centro Pluridisciplinar de Pesquisas Químicas, Biológicas e Agrícolas, Paulínea, SP, Brasil. 
colunas contendo dois tipos de solo. As colunas foram preenchidas com amostras de solo com e sem perturbação (desagregação) para cada tipo de solo, solo Bagan Datoh (argiloso) e o solo Labu (areno-argiloso) e foram observadas com relação à lixiviação-do pesticida durante 14 dias de umedecimento. $\mathrm{O}$ estudo mostrou que ocorreu menos lixiviação nas colunas de solo com alto teor de matéria orgânica, tal como o solo Bagan Datoh e especialmente em solos perturbados, onde a matéria orgânica foi homogeneamente misturada em todas as camadas.

Este trabalho é um dos poucos estudos existentes para avaliação do uso de carbofurano e o meio ambiente em São Paulo. Foi escolhida uma propriedade agrícola eáreas adjacentes (inclusiveno sedimento da margem do Rio Paraíba doSul) a esta propriedade do Distrito de Quiririm, que apresenta como atividade econômica principal o cultivo de arroz. Quiririm faz parte do Município de Taubaté, Vale do Paraíba, São Paulo, Brasil, latitude -23.0254 e longitude -45.6317, aproximadas (GoOGLE MAPS, 2010).

A propriedaderural investigada apresentava área cultivada de 230 hectares, sendo a principal praga da cultura o gorgulho aquático. Esta é uma pragachave para a cultura e o carbofurano é empregado há vários anos na região para o seu controle.

Durante o estudo, o produto comercial de nome Ralzer 50 GR (Fersol Indústria e Comércio S.A.), foi aplicado com uma pequena semeadora, que produz efeitos como se fosse aplicação a lanço. A dose aplicada de Ralzer 50 GR foi de $210 \mathrm{~g} /$ tabuleiro de 700 $\mathrm{m}^{2}$, dose que o proprietário rural decidiu e concluiu ser suficiente. Esta prática agrícola é amplamente utilizada pelos agricultores da região, o Ralzer é formulado com $5 \%$ de carbofurano, correspondendo a aproximadamente $10,5 \mathrm{~g}$ decarbofurano distribuidos em cada tabuleiro.

As amostras de solo e sedimento foram coletadas, em sete pontos previamente demarcados. Ponto 1 - Tabuleiro - amostras foram coletadas no tabuleiro com uma área de $700 \mathrm{~m}^{2}$ e que recebeu aplicação do carbofurano (de 3,0 a 3,5 kg do produto comercial/ ha). Ponto 2 - Valeta mestra - ( $2^{\mathrm{a}}$ comporta) que é um canal aberto e sem revestimento com 1,5 a 2,0 $\mathrm{m}$ de largura e profundidade de aproximadamente $2 \mathrm{~m}$, esta valeta drena água equivalente a aproximadamente 50 hectares de tabuleiros e recebe água dos tabuleiros da propriedade rural avaliada que possui cinco valetas mestra. Ponto 3 - Dreno A - este canal é aberto e sem revestimento, com dimensões de aproximadamente 3,0 × 3,0 m e recebe água de aproximadamente 200 hectares de tabuleiros, pertencentes a 3 propriedades rurais. Ponto 4 - Ponte do Chará, este ponto está situado a jusante do Dreno A, pelo ponto 4 também passa água de aproximadamente 200 hectares de tabuleiros, pertencentes a 3 propriedades rurais. Ponto 5 - localizado a 100 $\mathrm{m}$ à montante da desembocadura do dreno no rio Paraíba do Sul, traz água de tabuleiros de várias propriedades rurais. Ponto 6 - desembocadura do dreno no rio Paraíba do Sul, o ponto de coleta de sedimento é no rio Paraíba do Sul, onde deságua o dreno que traz água de várias propriedades. Ponto 7 - a 100 m à jusante da desembocadura do dreno A no rio Paraíba do Sul, neste ponto a coleta do sedimento deu-se na margem do rio.

Durante o período de1999/2000, foram coletadas amostras nas seguintes datas: 6/9/1999 (0 - dias após o tratamento - dat); 4/10/1999 (5 dat); 9/12/1999 (71 dat); 11/1/2000 (104 dat) e 4/5/2000 (218 dat), destacando-se que no ano 2000 foram realizadas apenas duas coletas, já que tanto as amostras de solo superficial quanto as de sedimento coletadas anteriormente em 0 dat, 5 dat e 71 dat não haviam sido detectados resíduos de carbofurano e de seu metabólito.

Aproximadamente $1 \mathrm{~kg}$ de solo e $1 \mathrm{~kg}$ de sedimento foram coletados em cada um dos 7 pontos, respectivamente, na camada superficial $(0$ a $10 \mathrm{~cm}$ aproximadamente) e na lateral dos drenos, sendo que a $1^{a}$ coleta foi antes da aplicação do agrotóxico e as demais depois da aplicação do inseticida. As amostras foram armazenadas sob refrigeração a $4^{\circ}$ C até a etapa de extração.

As amostras de solo e sedimento foram analisadas no Laboratório de Resíduos da Embrapa Meio Ambiente para determinar a presença de resíduos de carbofurano e de seu metabólito 3-hidroxicarbofurano, utilizando o método oficial, EPAUS method 8318 adaptado e validado pelo Laboratório de Resíduos de Pesticidas da Embrapa Meio Ambiente (Revisão 0, 1994).

Amostras de aproximadamente $1 \mathrm{~kg}$ de solo ou sedimento foram homogeneizadas e secas em bancadas do laboratório, previamente descontaminadas, por $24 \mathrm{~h}$. A seguir, foram peneiradas em tamis de malha de ferro de $2 \mathrm{~mm}$. De cada amostra inicial peneirada, retirou-se uma alíquota de $20 \mathrm{~g}$ de solo ou sedimento. Procedeu-se a pesagem de $20 \mathrm{~g}$ de solo em erlenmeyer de $250 \mathrm{~mL}$, adicionando-se a seguir $50 \mathrm{~mL}$ de acetonitrila. Posteriormente, as amostras foram colocadas no agitador mecânico por 2 horas. Em seguida, deixou-se o material em repouso por 5 a 10 minutos e retirou-se o sobrenadante, transferindo para um tubo de centrífuga. Repetiu-se a extração por mais duas vezes utilizando $20 \mathrm{~mL}$ de acetonitrila ao solo ou ao sedimento, estes foram colocados sob agitação por 1 hora, seguido de repouso por 5 a 10 minutos, retirando-se o sobrenadante. Os 3 extratos foram misturados e centrifugados por 10 minutos a 2.000 rotações por minuto (RPM). O sobrenadantefoi decantado, diluído com acetonitrila e alíquota de 10 $\mathrm{mL}$ foi retirada com auxílio de pipeta volumétrica, e 
transferida para um tubo de ensaio de $10 \mathrm{~mL}$. Ela foi concentrada com corrente suave de $\mathrm{N}_{2}$ até perfazer o volume de $0,9 \mathrm{~mL}$. Ao extrato adicionou-se $1,1 \mathrm{~mL}$ de água, suficiente para reconstituir a proporção da fase móvel para análise por HPLC.

A verificação do desempenho do método foi feita com o emprego de amostras fortificadas na concentração 1,25 $\mu \mathrm{g} \cdot \mathrm{kg}^{-1}$ e $2,5 \mu \mathrm{g} \cdot \mathrm{kg}^{-1}$ (dose e o dobro da dose recomendada), porém, ela não foi considerada para a avaliação do método, por não ser representativa. Os limites de detecção (LD) e de quantificação (LQ) foram calculados com o emprego de curva de calibração e adotando-se o valor teórico de 3 vezes o desvio padrão das injeções para a determinação do LD e 5 vezes o desvio padrão para a determinação do LQ, desta forma o menor nível de detecção para os compostos analisado é de $0,45 \mu \mathrm{g} \cdot \mathrm{kg}^{-1}$ para o 3-hidroxicarbofurano e de $0,37 \mu \mathrm{g} . \mathrm{kg}^{-1}$ para o carbofurano. Os resultados obtidos foram comparados com as injeções do padrão analítico puros.

As amostras foram analisadas com o uso da cromatografia líquida de alta eficiência (HPLC ou CLAE), equipamento utilizado foi da marca Shimadzu, com detector de fluorescência RF-535. Para a separação dos compostos foi utilizada uma coluna cromatográfica de fase reversa, $\mathrm{C} 18$ Microsorb MV ${ }^{\mathrm{T}}$ M100Aa Varian $5 \mu \mathrm{m}$, de tamanho 4,6 $250 \mathrm{~mm}$. A fase móvel empregada na análise foi acetonitrila/água na proporção de $45 / 55$ e a uma vazão de 1,0 mL.min ${ }^{-1}$. Para a identificação do inseticida carbamato e metabólito utilizou-se o composto orto-ftaldialdeído, para promover a reação em pós-coluna. O detetor de fluorescência foi ajustado para l excitação $455 \mathrm{~nm}$ e 1 emissão $340 \mathrm{~nm}$ e volume injetado de $50 \mu \mathrm{L}$. As quantidades nas amostras foram calculadas a partir das curvas de calibração construídas com pares de concentração $x$ resposta de soluções de padrão de carbofurano e 3- hidroxicarbofurano (soluções preparadas com padrão analítico de carbofurano $99,7 \%$ de pureza e 3-hidroxicarbofuran 98,5\% de pureza, Dr. Ehrenstorfer, Alemanha).

Os estudos de fortificação foram baseados na dose especificada na bula do produto, porém, devese salientar que devido à prática agrícola do local, os agricultores, inclusive aquele da área escolhida para o desenvolvimento do estudo, utilizavam valores $1 / 5$ da dose recomendada para o controle de O. oryzae. Desta forma, os limites de quantificação e detecção ficaram acima do valor esperado de $0,21 \mu \mathrm{g} \cdot \mathrm{kg}^{-1}$ do inseticida em amostra de solo, fato que deve ser destacado é que a cultura de arroz é amplamente difundida na região podendo haver resíduos remanescentes de plantações anteriores e aqueles provenientes do meio ambiente. Este estudo avaliou as condições reais de campo e de aplicação do inseticida carbofurano na região, portanto, não é um estudo experimental, onde as condições de campo são previamente escolhidas e monitoradas durante o estudo.

Como mencionado anteriormente, a dose utilizada no período foi de aproximadamente $1 / 5 \mathrm{da}$ dose recomendada pelo fabricante, fato este que pode levar à conclusão de que os resíduos do carbofurano puderam ter ficado abaixo do limite de detecção, porém, poderia existir resíduo de aplicações anteriores, já que, para o carbofurano no solo, a fotodecomposição não é uma importante rota de degradação, de acordo com a Organização Mundial deSaúde(WorLD..., 1996). Portanto, mesmo tendo-se detectado a ausência de carbofurano neste estudo, não é possível concluir que em solo superficial (até $10 \mathrm{~cm}$ aproximadamente) o carbofurano teria sido degradado pela luz solar. Ainda, de acordo com WorlD... (1996) onde descreve que foram realizadas observações, que repetidas aplicações de carbofurano não resultaram em acúmulo de resíduos, atribuindo a isto a degradação química e microbiológica do carbofurano, principalmente por hidrólise e hidroxilação.

XIANG-YANG et al. (2009) observaram que a biomassa carbonizada, em estudo utilizando a combustão do eucalipto por combustão incompleta, mostrou ser particularmente efetiva para sorção e para sequestrar contaminantes orgânicos no solo devido a sua grande área superficial, alta microporosidade e outras propriedades físico-químicas. Em relação à dissipação de agrotóxicos, a partir de solos não compensadosa, após a carbonização da biomassa, a perda de clorpirifós e carbofurano não foi corrigida.

De acordo com International Union of Pure and Applied Chemistry (IUPAC, 2012), o índice de GUS para a lixiviação do carbofurano em solo é de meia vida de carbofurano no intervalo de 5 a 30 dias, o mesmo foi observado por TomLIN (1994) que relatou que a meia vida de carbofurano em solo é de 30 a 60 dias.

SILva et al.(2009) detectaram o carbofurano em todas as regiões orizícolas no Sul do Brasil. Os autores relataram a alta solubilidade em água e ao baixo coeficiente de adsorção ao solo, Kow $=1,52$, a $20^{\circ} \mathrm{C}$. O carbofurano possui alto potencial de ser transportado quando dissolvido em água e assim contaminar os mananciais hídricos superficiais.

A prática agrícola da subdose pode ter favorecido a não determinação do agrotóxico. Deve-se salientar que a sub-dosagem pode contribuir para a resistência dos insetos nocivos.

\section{AGRADECIMENTOS}

À FAPESP pela concessão de Auxílio à Pesquisa pela importante contribuição para o desenvolvimento da Dissertação de Mestrado do primeiro autor 
intitulada: Impactos ao Ambiente e Riscos Potenciais à Saúde, Decorrentes do Uso de Carbofuran em Área de Produção de Arroz, Taubaté, São Paulo, apresentada à Faculdade de Saúde Publica da USP , em 2001. Aos colegas Teresa Jocys pela sugestão do tema e apoio e Minoru Takada pela escolha da propriedade rural a ser estudada e sugestões e à química Lourde Silvestre de Souza pela valiosa colaboração no Laboratório de Resíduos da Embrapa Meio Ambiente nas análises.

\section{REFERÊNCIAS}

BRASIL. Ministério da Agricultura, Pecuaria e Abastecimento. Agrofit: Sistema de Agrotóxicos Fitossanitários. Brasília: MAPA, 2003. [online] Disponível em: <http:/ / agrofit.agricultura.gov.br/agrofit_cons/principal_ agrofit_cons>. Acesso em: 13 abr. 2010.

FARAHANI, G.H.N.; SAHID, I.B.; ZACARIA, Z.; KUNTOM, A.; OMAR, D. Study on the downard movement of carbofuran in two malaysian soils. Bulletin of Environmental Contamination and Toxicology, v.81, n.3, p.294-298, 2008.

GOOGLE MAPS. Mapa da região de estudo. Disponível em: <http:/ / maps.google.com.br/maps>. Acesso em: 23 abr. 2010.

IUPAC - International Union of Pure and Applied Chemistry. Footprint pesticides properties database. Disponível em: <http:/ /.sitem.herts.ac.uk/aeru/iupac>. Acesso em: 6 nov. 2012.

JINHE, S.; JIIANYING, G.; ZIYUAN, C. Fate of carbofuran in model rice/fish. Ecosystems. Pesticide Science, v.26, p.147-157, 1989.

JURY, W.A.; GHODRATI, M. Overview of organic chemical environmental fate and transport modeling approaches. In: SAWHNEY, B.L.; BROWN, K. (Ed.). Reactions and movement of organic chemicals in soils. Madison, WI, 1989. p.271-304.

LALAH, J.O.; WANDIGA, S.O. Adsorption/desorption and mobility of carbofuran in soil samples from Kenya. Bulletin of Environmental Contamination and Toxicology, v.56, p.575-583, 1996.

WORLD HEALTH ORGANIZATION. Guidelines for drinking-water quality v.2, Health criteria and other supporting information. Geneva: WHO, 1996.

SILVA, D.R.O.; AVILA, L.A.; AGOSTINETTO D.; MAGRO, T.D.; OLIVEIRA, E.; ZANELLA, R.; NOLDIN, J.A. Monitoramento de agrotóxicos em águas superficiais de regiões orizícolas no sul do Brasil. Ciência Rural, v.39. n.9, p.2383-2389, 2009.

TOMLIN, C.D.S. Carbofuran the pesticide manual. In: (Ed.). Carbofuran. 10.ed. Cambridge: British

Crop Protection Council, 1994. p.152-153.

TRABUE, S.L.; FENG X.; OGRAN, A.V.; OU, L.T. Carbofuran degradation in soil profiles. Journal of Environmental Science and Health Part B, v.32, n.6, p.861-878, 1997.

UNITED STATES. Environmental Protection Agency. National Primary Drinking Water Regulations: Carbofuran. Method 8318. Revision 0, 1994. Washington, DC: EPA, 1995.

XIANG-YANG, Y.; GUANG-YING, Y.; KOOKANA, R.S. Reduced plant uptake of pesticides with biochar additions to soil. Chemosphere, v.76, p.665-671, 2009.

Recebido em $1 / 9 / 11$

Aceito em 9/11/12 